\title{
ANÁLISE DA FUNÇÃO RESPIRATÓRIA EM PACIENTES OBESOS SUBMETIDOS À OPERAÇÃO FOBI-CAPELLA
}

\author{
RESPIRATORY FUNCTION IN OBESE PATIENTS SUBMITTED TO FOBI-CAPELLA \\ OPERATION
}

\author{
Áurea Maria Oliveira da Silva ${ }^{1}$; Ilka de Fátima Santana Boin, ACBC-SP²; \\ José Carlos Pareja, ECBC-SP²; Luis Alberto Magna ${ }^{3}$
}

\begin{abstract}
RESUMO: Introdução: O objetivo deste trabalho foi mensurar quantitativamente essas alterações respiratórias desses pacientes comparando-os até 30 dias de pós-operatório. Método: Foram realizadas avaliações respiratórias nos períodos pré e pós-operatório de cirurgia bariátrica em obesos mórbidos com IMC superior a $39 \mathrm{~kg} / \mathrm{m}^{2}$, através de gasometria arterial, prova de função respiratória, manovacuômetria, incentivador da respiração e cirtometrias. Foram realizadas também orientações fisioterápicas respiratórias e tratamento no pós-operatório, com dados comparativos entre as avaliações feitas no préoperatório, no $1^{\circ}, 14^{\circ}$ e $30^{\circ}$ dia pós-operatórios. Resultados: Até o $30^{\circ}$ dia de pós-operatório, esses indivíduos não obtiveram diferença significativa nos parâmetros estudados, não havendo, em decorrência do tratamento fisioterápico, complicações respiratórias. Conclusão: Não houve alterações dos parâmetros analisados, nem complicações respiratórias neste estudo com intervenção fisioterápica pré e pós-operatório de cirurgia bariátrica. Estudos devem ser realizados, para mensuração de um tempo maior de pós-operatório e de exercícios específicos, podendo, assim apresentar resultados diferentes (Rev. Col. Bras. Cir. 2007; 34(5): 314-320).
\end{abstract}

Descritores: Fisioterapia; Obesidade; Cirurgia bariátrica; Espirometria; Testes de função respiratória.

\section{INTRODUÇÃO}

A obesidade extrema e a síndrome cardiopulmonar que a acompanha já era conhecida na década de $50^{1}$. É sabido que a obesidade mórbida pode promover uma síndrome restritiva pelo acúmulo de gordura peritorácica e abdominal diminuindo os volumes pulmonares ${ }^{2}$, o volume de reserva expiratório (VRE) e a capacidade residual funcional (CRF), promovendo também anomalias na ventilação/perfusão pela hipoxemia de repouso e de decúbito dorsal provavelmente devido ao fechamento de pequenas vias aéreas observado neste tipo de doente.

A maior parte das complicações respiratórias no pósoperatório decorre de causas pré-operatórias, como doença prévia pulmonar; gasometria arterial alterada; capacidades, volumes e fluxos alterados e no intra-operatório pelas ventilações inadequadas, o tempo cirúrgico prolongado, abertura abdominal, aumento do tempo de ventilação mecânica, aumento do tempo de imobilização e outras ${ }^{3-6}$.

Assim vimos a necessidade de verificar as alterações e modificações desses pacientes após uma perda de peso significativa no pós-operatório de cirurgia bariátrica, por um período definido de até 30 dias, para se detectar possíveis complicações pulmonares, bem como as suas alterações, levando-se em conta a atuação fisioterápica.

\section{MÉTODO}

Foram avaliados 50 pacientes que seriam submetidos à cirurgia de Fobi-Capella ${ }^{7}$ no Hospital de Clínicas da Universidade Estadual de Campinas (HC/UNICAMP), no período de março de 2000 a março de 2002.

Estes pacientes possuíam risco médio ou baixo de complicações respiratórias, analisadas pela equipe da Disciplina de Pneumologia da Faculdade de Ciências Médicas (FCM/UNICAMP), sob os critérios da escala de Torrington e Henderson (1988) e índice de massa corpórea (IMC) ${ }^{3} 39$. Foram considerados critérios de exclusão: doença gástrica que impedisse a operação e doença cardíaca grave.

Os pacientes foram relacionados em uma lista de espera e orientados pela fisioterapeuta ao aprendizado do uso do incentivador da respiração, da necessidade de exercícios diários ativos, como caminhadas e hidroginástica e de explicações gerais sobre a operação e o pós-operatório.

Antes de qualquer procedimento, os pacientes foram informados a que tipos de tratamento seriam submetidos e assinavam um termo de consentimento que foi previamente aprovado pelo Comitê de Ética em Pesquisa da FCM/ UNICAMP.

No período de internação, os pacientes foram submetidos à fisioterapia convencional durante o pré-operatório

1. Fisioterapeuta Mestre da Faculdade de Ciências Médicas - UNICAMP.

2. Professor Doutor do Departamento de Cirurgia Faculdade de Ciências Médicas - UNICAMP

3. Professor Titular do Departamento de Genética Médica da Faculdade de Ciências Médicas / UNICAMP

Recebido em 19/01/2007

Aceito para publicação em 20/03/2007

Conflito de interesses: nenhum

Fonte de financiamento: nenhuma

Trabalho realizado no Hospital de Clínicas da Universidade Estadual de Campinas (HC/UNICAMP). 
até o momento da alta hospitalar, de acordo com a clínica apresentada, através das manobras fisioterápicas tradicionais, exercícios ativos, ativos-assistidos e deambulação.

Após alta hospitalar os pacientes eram orientados à realização do incentivador da respiração, bem como a caminhadas diárias conforme possibilidade individual, aumentando gradativamente o tempo e espaço percorrido sob a forma de condicionamento físico.

Foram avaliadas as medidas antropométricas: cintura/quadril e pescoço em cm (utilizando-se duas fitas métricas de 1,5 cm unidas). Para avaliação respiratória foram avaliados em quatro momentos específicos: uma vez no pré-operatório (I), e três vezes no pós-operatório, sendo a primeira no pósoperatório um (PO1), 14 dias após o ato cirúrgico (PO14) e 30 dias após o ato cirúrgico (PO30), conforme esquema da tabela 1, através dos seguintes exames: radiograma de tórax, prova de função pulmonar, gasometria arterial, manovacuômetria, incentivador da respiração e cintometria.

Espirometria: foram realizados os testes de espirometria no laboratório da Pneumologia do HC/UNICAMP, com o aparelho da ANAMED 4.000PC (programa Breeze), com a supervisão das técnicas do Serviço. Os pacientes se mantiveram na posição sentada para a execução do teste. Este teste foi utilizado para se verificar o grau de restrição, obstrução ou perda elástica pulmonar, segundo a avaliação do pneumologista responsável. Foram anotados os seguintes itens expressos em \% ou valor absoluto: Capacidade Vital Forçada (CVF); Volume Expiratório Forçado no primeiro segundo $\left(\mathrm{VEF}_{1}\right)$; Fluxo Expiratório Forçado em 25\% (FEF 25\%); Fluxo Expiratório Forçado Máximo (FEF max); Ventilação Voluntária Máxima(VVM).

Gasometria Arterial Simples: foram coletadas amostras de sangue da artéria radial, com o paciente na posição sentada ou deitada, sendo analisados, com o aparelho Bionova, os seguintes ítens: pressão parcial de oxigênio $\left(\mathrm{pO}_{2}\right)$; pressão parcial de dióxido de carbono $\left(\mathrm{pCO}_{2}\right)$ e porcentagem de saturação de oxigênio $\left(\mathrm{Sat}_{2}\right)$.

Manovacuômetria: foi mensurada com o aparelho manovacuômetro da marca Ger-ar modelo MV-300, de variação de $-300 \mathrm{a}+300 \mathrm{~cm} \mathrm{H_{2 }} \mathrm{O}$, onde se mediam as pressões positivas (expiratórias - manômetro) e negativas (inspiratórias - vacuômetro), isto é, mediu-se a força dos músculos respiratórios. Os pacientes se mantinham na posição sentada sem o inibidor nasal e eram orientados a fazer primeiramente três expirações forçadas em esforço máximo e após três inspirações forçadas em esforço máximo e eram anotados os resultados melhores dos três procedimentos. Foram anotados os seguintes valores: pressão inspiratória máxima (PIM) e pressão expiratória máxima (PEM):

Incentivador da Inspiração: foi utilizado o aparelho individual marca Respiron®. Os pacientes eram orientados a adquirir e treinar antes da operação, fazendo com que esse treinamento consistisse de no mínimo cinco séries de dez vezes, três vezes ao dia, durante todo o período pré-operatório, intensificando-se no momento da internação e aumentando o grau de dificuldade gradativamente. Os valores obtidos foram somente anotados de forma individual e qualitativa visual, a fim de se observar a melhora ou piora do estado respiratório em cada fase, comparativamente. Esses valores foram relacionados, para a análise estatística, em uma escala crescente de valores identificados pelo grau de dificuldade encontrada. Assim o paciente que não conseguia elevar nenhuma esfera no menor grau de dificuldade pontuava 0 até aquele que elevava as três esferas com o maior grau de dificuldade, pontuava 14.

Após a operação foram anotados os dados intraoperatórios como: data da operação; técnica utilizada, tempo de procedimento (em minutos), tipo de incisão realizada e o tempo de internação no hospital (em dias). Foram anotadas, também as complicações gerais, respiratórias ou vasculares e o tempo em que ocorreram.

Os dados estatísticos foram analisados pelo método de Friedman, quando se tratava de três variáveis ou mais e o método de Wilcoxon foi utilizado quando se tratava de duas variáveis, os dois com um intervalo de confiança de $95 \%$.

\section{RESULTADOS}

Foram estudados 39 mulheres (78\%) e 11 homens (22\%). O resultado de outras variáveis consta na tabela 2. Dentre os pacientes estudados, 19 (38\%) pacientes eram tabagistas ou ex-tabagistas.Todos os dados obtidos dos exames de prova de função pulmonar, gasometria arterial simples, manovacuômetria, cirtometrias e incentivador da

Tabela 1 - Esquema de acompanhamento dos períodos de estudo e das provas realizadas para a avaliação respiratória.

\begin{tabular}{lcccccc}
\hline & RX & PFP & Gaso. & Manov. & Incent. & Cirtometria \\
\hline Pré-operatório & $\mathrm{X}$ & $\mathrm{X}$ & $\mathrm{X}$ & $\mathrm{X}$ & $\mathrm{X}$ & $\mathrm{X}$ \\
PO 1 & & $\mathrm{X}$ & $\mathrm{X}$ & $\mathrm{X}$ & $\mathrm{X}$ & \\
PO14 & & $\mathrm{X}$ & $\mathrm{X}$ & $\mathrm{X}$ & $\mathrm{X}$ & \\
PO30 & & & & $\mathrm{X}$ & $\mathrm{X}$ \\
\hline
\end{tabular}

$R X$ - radiograma do tórax;

PFP-espirometria;

Gaso - gasometria arterial;

Manov. - manovacuômetria;

Incent. - incentivador da inspiração;

PO 1 - primeiro dia de pós-operatório;

PO14 - décimo quarto dia de pós-operatório;

PO30 - trigésimo dia de pós-operatório. 
Tabela 2 - Resultado das variáveis encontradas no estudo no período pré-operatório.

\begin{tabular}{|c|c|c|c|c|}
\hline \multirow[b]{2}{*}{ IDADE (anos) } & \multicolumn{2}{|c|}{ Média } & \multicolumn{2}{|c|}{ Variação (minimo/máximo) } \\
\hline & 36,2 & $( \pm 8,03)$ & 19 & 60 \\
\hline PESO $(\mathrm{Kg})$ & 152,6 & $( \pm 34,4)$ & 95 & 218 \\
\hline $\operatorname{ALTURA}(\mathrm{m})$ & 1,64 & $( \pm 0,08)$ & $1,47 \mathrm{e}$ & 1,83 \\
\hline $\mathrm{IMC}\left(\mathrm{Kg} / \mathrm{m}^{2}\right)$ & 56,44 & $( \pm 10,61)$ & $39,06 \mathrm{e}$ & 79,24 \\
\hline Tempo em obesidade (anos) & 18,46 & $( \pm 11,15)$ & 2 & 51 \\
\hline
\end{tabular}

respiração, foram analisados de acordo com a realização das provas solicitadas nos momentos analisados, sem prejuízo da confiança estatística, pois os pacientes foram descartados de modo aleatório, pela impossibilidade de realização dos testes, seja pela ausência deste paciente ou pela impossibilidade técnica (aparelho quebrado ou amostra inadequada).

Somente 30 dos 50 pacientes do estudo, possibilitaram a verificação das medidas antropométricas (Tabela 3 ).

$\mathrm{Na}$ avaliação radiológica anterior ao procedimento cirúrgico, observou-se que: 18 (36\%) pacientes possuíam elevação da cúpula diafragmática direita, somente um (2\%) paciente possuía a cúpula diafragmática esquerda elevada e somente um (2\%) paciente possuía uma opacidade em base direita e os outros 30 pacientes $(60 \%)$ tiveram seus radiogramas normais.

Das incisões realizadas, observou-se somente duas (4\%) incisões subcostais sendo os outros 48 (96\%) dos pacientes tiveram suas incisões medianas supra-umbilicais. $\mathrm{O}$ tempo cirúrgico variou de 120 a 360 minutos, tendo-se como média 187,6 minutos $( \pm 43,77)$; O tempo de internação médio foi de 5,81 dias $( \pm 1,39)$, variando entre 4 e 10 dias, com desvio padrão de 1,39 .

Dos pacientes estudados 35 (70\%) tiveram algum tipo de complicação, salientando que somente um teve complicação respiratória no período de internação devido a um derrame pleural infectado. As complicações gerais observadas, mas não anotadas quanto a porcentagem, foram: febre, diarréia e não aceitação da ingesta via oral da alimentação proposta.
Somente um paciente teve reinternação porque apresentou pneumonia e evisceração. Dois $(4 \%)$ pacientes morreram ainda no período de internação em decorrência de complicações pós-operatórias de origem não pulmonar.

Também foi verificada uma perda de peso, sem a média antes da cirurgia de $152,58 \mathrm{Kg}$ e a média no $30^{\circ}$ dia de pósoperatório de $140,84 \mathrm{Kg}$.

A espirometria foi observada em 26 pacientes nos tempos pré-operatório (CVFI), 14 dias (CVF14) e 30 dias (CVF30) de pós-operatório, sendo que os valores obtidos não mostraram diferença significativa entre eles (Tabela 4).

Esses mesmos pacientes $(n=20)$ foram estudados nesses dados por valores absolutos e analisados estatisticamente para se obter maior confiabilidade dos resultados, visto que os pacientes possuíam pesos superiores à capacidade da balança do serviço em que foram realizados os exames de espirometria. (Tabela 5).

A gasometria arterial foi realizada em todos os pacientes, sendo que somente 36 pacientes dos 50 pacientes estudados a realizaram em todos os momentos observados na Tabela 6. Somente mostrou-se significativo no $\mathrm{PO}_{1}$ do parâmetro $\mathrm{pCO}_{2}$, sendo que a diferença é identificada quando esta variável é retirada e as demais são comparadas.

A manovacuômetria com 29 pacientes que compareceram nos quatro momentos, sendo que os dados descartados não comprometeram os resultados finais pelo descarte aleatório. A comparação das médias de PIM mostrou haver diferença significativa entre elas $(\mathrm{p}<0,001)$; mas esta diferen-

Tabela 3 - Média e desvio padrão das medidas antropométricas nos momentos: pré-operatório e no $30^{\circ}$ dias após a cirurgia bariátrica.

\begin{tabular}{lcccc}
\hline Cirtometrias & \multicolumn{2}{c}{ Pré-operatório } & \multicolumn{2}{c}{ Pós-operatório de 30 dias } \\
\hline Cintura $(\mathrm{cm})$ & 137,58 & $( \pm 18,17)$ & 131,41 & $( \pm 17,12)$ \\
Quadril $(\mathrm{cm})$ & 159 & $( \pm 19,04)$ & 153,05 & $( \pm 18,45)$ \\
Circunferência do pescoço $(\mathrm{cm})$ & 46,30 & $( \pm 4,10)$ & 43,71 & $( \pm 3,92)$ \\
\hline
\end{tabular}

Tabela 4 - Resultados das médias em valores percentuais do pré-operatório até 30 dias de pós-operatório da espirometria.

\begin{tabular}{lrlrl}
\hline & Pré-operatório & 14 dias pós-op & 30 dias pós-op & \\
\hline $\mathrm{CVF} \%$ & $93,10 \pm 13,77$ & $88,79 \pm 14,97$ & $93,85 \pm 15,57$ & $\mathrm{p}=0,11$ \\
$\mathrm{VEF}_{1} \%$ & $95,98 \pm 15,14$ & $88,69 \pm 18,20$ & $95,03 \pm 19$ & $\mathrm{p}=0,26$ \\
$\mathrm{FEF}_{25 \%} \%$ & $99,69 \pm 25,64$ & $88,76 \pm 27,74$ & $96,06 \pm 20,43$ & $\mathrm{p}=0,62$ \\
$\mathrm{FEF}$ máx $\%_{\text {VVM\% }}$ & $105,65 \pm 26,31$ & $94,03 \pm 26,31$ & $102,58 \pm 27,30$ & $\mathrm{p}=0,70$ \\
\hline
\end{tabular}


ça devido ao valor da média desta variável no primeiro dia de pós-operatório e que esta significância desaparece quando as demais são comparadas ( $\mathrm{p}=0,36)$; e no PEM, a comparação destas médias, mostrou haver diferença significativa entre elas ( $\mathrm{p}<0,001$ ); sendo que esta significância não é relevante, pois se retirarmos os valores da média no primeiro dia de pós-operatório $(\mathrm{p}<0,001)$ e do resultado inicial $(\mathrm{p}=0,62)$, desaparece esta significância, sendo a comparação de relação em melhora, estatisticamente do valor inicial ao valor final de 30 dias de pós-operatório tendo um p=0,001 (Tabela 6).

No parâmetro incentivador da respiração RESPIRONâ - foram observados os resultados de 23 pacientes obtendo-se os seguintes resultados: no pré-operatório a média foi de $9,52( \pm 3,81)$; no primeiro dia de pós-operatório, a média foi de $3,22( \pm 1,24)$; 'no décimo-quarto dia de pós-operatório foi de $6,91( \pm 3,85)$ e no trigésimo dia de pós-operatório foi de $10,13( \pm 3,15)$. Na comparação destas médias, houve diferença significativa entre elas $(\mathrm{p}<0,001)$; sendo que esta significância desaparece se retirarmos os valores da média no primeiro dia de pós-operatório.

\section{DISCUSSÃo}

Em 1990 o American College of Physicians demonstrou após vários artigos um consenso de indicações e testes respiratórios pré-operatórios. Nas internações cirúrgicas no andar superior do abdome, a indicação foi de que a espirometria tem sido o teste de eleição para se detectar um fator de risco pulmonar pós-operatório para este tipo de operação, bem como prudentemente a análise da gasometria arterial (principalmente em pacientes com história de tabagismo e dispnéia). A utilização no pós-operatório de pressão positiva (CPAP) e incentivadores da respiração diminuem as complicações pós- operatórias e a longa permanência hospitalar.

Os pacientes obesos mórbidos que perdem peso, como por exemplo: pacientes que diminuem o IMC de 50 para $37 \mathrm{~kg} / \mathrm{m}^{2}$, têem um aumento de $75 \%$ no volume de reserva expiratória, $25 \%$ de melhora no volume residual e na capacidade funcional residual e $10 \%$ de melhora na ventilação voluntária máxima ${ }^{8}$.

Nossos dados somente evidenciaram um aumento significativo na capacidade vital, pois nos outros parâmetros a melhora não foi significativa (capacidade pulmonar total e $\left.\mathrm{VEF}_{1} / \mathrm{CVF}\right)$.

No nosso estudo, os pacientes possuíam alterações que durante o período do estudo (30 dias) não se modificaram significativamente. Por outro lado, o VVM foi adotado como parâmetro pré-operatório como um valor de probabilidade de complicação direta ou indireta da reserva respiratória, visto que muitos pacientes relatavam dispnéia aos esforços pela excessiva massa corpórea e que após essa perda, essa dispnéia desaparece comparativamente ao período pré-operatório relatada pelos pacientes. Salientamos que dos 50 pacientes do estudo, somente sete possuíam a espirometria fora dos padrões de normalidade, isto é, possuíam de leve a moderado grau de restrição ${ }^{9-12}$.

Observou-se uma melhora dos padrões respiratórios em pacientes submetidos à operação bariátrica com alterações obstrutivas respiratórias após 180 dias de pós-operatório.

É importante ressaltar que somente alguns dados possuíam significância estatística de melhora: a capacidade vital forçada, a capacidade pulmonar total, a capacidade funcional residual, o volume residual, e o volume de reserva expiratória. Estes dados foram melhorados provavelmente pelo aumento no "endurance" isocinético muscular e atividade da síntese de glicogênio?.

Table 5 - Resultados da espirometria em valores absolutos.

\begin{tabular}{lrrrrr}
\hline & Initial & Day 14 & Day 30 & \\
\hline $\mathrm{FVC}$ & $3.59 \pm 1.11$ & $3.50 \pm 1.01$ & $3.66 \pm 1.02$ & $\mathrm{p}=0.25$ \\
$\mathrm{FEV}_{1}$ & $2.96 \pm 0.84$ & $2.95 \pm 0.93$ & $3.04 \pm 0.87$ & $\mathrm{p}=0.77$ \\
$\mathrm{FEF}_{25 \%}$ & $6.06 \pm 2.08$ & $5.97 \pm 1.99$ & $6.34 \pm 1.85$ & $\mathrm{p}=0.52$ \\
$\mathrm{FEF}_{\text {Max }}$ & $7.04 \pm 2.46$ & $6.65 \pm 2.20$ & $7.19 \pm 2.25$ & $\mathrm{p}=0.96$ \\
$\mathrm{MVV}$ & $94.75 \pm 35.82$ & $98.75 \pm 27.26$ & $105.80 \pm 31.91$ & $\mathrm{p}=0.26$ \\
\hline
\end{tabular}

Tabela 6 - Resultados das médias dos valores de gasometria arterial e da manovacuômetria do pré-operatório até 30 dias de pós-operatório (PO 1, PO 14 e PO 30).

\begin{tabular}{lccccc}
\hline & Inicial & $\mathbf{P O}_{\mathbf{1}}$ & $\mathbf{P O}_{14}$ & $\mathbf{P O}_{\mathbf{3 0}}$ & \\
\hline $\mathrm{PO}_{2}(\mathrm{mmHg})$ & $83,29 \pm 20,93$ & $79,38 \pm 34,61$ & $77,39 \pm 11,74$ & $80,63 \pm 21,91$ & $\mathrm{p}=0,52$ \\
$\mathrm{PCO}_{2}(\mathrm{mmHg})$ & $37,38 \pm 4,19$ & $39,99 \pm 5,83$ & $36,03 \pm 4,17$ & $36,39 \pm 4,03$ & $\mathrm{p}=<0,01^{*}$ \\
& & & & & $\mathrm{p}=0,44$ \\
$\mathrm{Sat}_{2}(\%)$ & $95,71 \pm 1,80$ & $93,76 \pm 5,19$ & $94,74 \pm 3,03$ & $94,26 \pm 5,17$ & $\mathrm{p}=0,95$ \\
$\mathrm{PIM}\left(\mathrm{cmH}_{2} \mathrm{O}\right)$ & $49,83 \pm 26,87$ & $18,97 \pm 20,06$ & $36,21 \pm 24,12$ & $44,48 \pm 21,64$ & $\mathrm{p}<0,001^{*}$ \\
& & & & & $\mathrm{p}=0,36$ \\
$\mathrm{PEM}\left(\mathrm{cmH}_{2} \mathrm{O}\right)$ & $93,62 \pm 40,81$ & $26,38 \pm 31,25$ & $51,03 \pm 32,66$ & $64,48 \pm 32,9$ & $\mathrm{p}<0,001^{* *}$ \\
& & & & & $\mathrm{p}=0,62$ \\
\hline
\end{tabular}


No estudo de De Lorenzo et al ${ }^{10}$ há referência à perda de peso corporal, sendo altamente ligada ao decréscimo da massa gorda total (tecido adiposo somente) e sem mudança significativa com a perda da massa magra total, com um "aperfeiçoamento" da função pulmonar em: capacidade vital, volume de reserva expiratória, volume expiratório forçado no primeiro segundo e fluxo expiratório forçado em $50 \%$ e de 25 a $75 \%$, esses dados foram encontrados em indivíduos que não possuíam evidencias de síndrome obstrutivas ${ }^{11}$. Neste estudo também houve uma grande significância de perda de peso corporal e o aumento da ventilação voluntária máxima, relacionando que pacientes que não perdiam em musculatura (com dieta ou cirurgia), melhoraram a prova de função pulmonar.

No trabalho de Ray ${ }^{12}$, foram comparados 29 pacientes, antes e depois de perderem peso, as funções respiratórias e verificadas as melhoras significativas na capacidade vital, no volume de reserva respiratória e na ventilação máxima voluntária, mas reduziram os valores de capacidade de difusão para o monóxido de carbono e não encontraram mudanças significativas nos valores da capacidade inspiratória, na capacidade pulmonar total, na capacidade funcional residual, no volume residual e no volume expiratório forçado no primeiro segundo/capacidade vital em \%. No mesmo estudo foi discutida a manobra da VVM, onde se diz que o aumento de peso pode ser a razão para a redução da capacidade de pacientes obesos para a performance na manobra de VVM, pela demanda rápida e profunda da respiração, sendo que nessa população a complacência da parede torácica é $1 / 3$ menor do que nos indivíduos normais, ocasionando um trabalho respiratório muito intenso identificado pela ventilação minuto ${ }^{12,13}$.

No presente estudo, verificou-se que não houve melhora significativa em nenhum dos parâmetros analisados, nos períodos de $14^{\circ}$ dia e $30^{\circ}$ dia de pós-operatório. Os dados foram verificados tanto em valores percentuais, quanto em valores absolutos, visto que o serviço utilizado não possuía balança adequada ao peso dos pacientes do estudo, podendo assim haver uma alteração dos valores.

A avaliação pré-operatória e a profilaxia pós-operatória são fundamentais para a diminuição da incidência de complicações pós-operatórias ${ }^{14}$. As atelectasias e a redução dos volumes pulmonares foram observados em 53\% dos pacientes deste estudo, comparado com outro de $9 \%$ de pacientes não obesos ${ }^{15}$. A posição em que se realiza o exame em obesos com IMC acima de $30 \mathrm{~kg} / \mathrm{m}^{2}$, sentado ou em posição ortostática não modifica de modo significativo os dados como nos indivíduos normais ${ }^{15}$, embora possa ser dito que o volume total aumentaria nos indivíduos obesos em posição ortostática ${ }^{16}$. Ali et $a l^{4}$, descreveram as alterações respiratórias do pós-operatório de cirurgia abdominal do andar superior: as atelectasias, as infecções pulmonares e hipoxemia arterial, sendo estas encontradas em até $70 \%$ do pós-operatório, sendo que a anestesia, o tipo de incisão e o tempo de operação influenciariam neste tipo de complicação, bem como sua condição respiratória no pré-operatório. Assim, o dado de hipoxemia foi um dos dados observados de complicação respiratória em pacientes obesos que são submetidos à cirurgia bariátrica: a tensão de oxigenação e/ou de retenção de $\mathrm{CO}_{2}$.
No presente estudo, estudou-se pacientes com IMC acima de $39 \mathrm{~kg} / \mathrm{m}^{2}$, sendo que $52 \%$ não relataram doença pulmonar prévia e $38 \%$ foram ou eram tabagistas, mesmo assim o índice de complicações respiratórias foi de $4 \%$.

Também o tempo cirúrgico em nosso estudo variou em média de 187,6 minutos $( \pm 43,77)$ e a incisão de escolha foi a xifo-umbilical, sendo de acordo com o descrito na literatura.

A melhora dos padrões gasométricos estão diretamente relacionados à perda de peso ${ }^{1,17}$, sugerindo que no pósoperatório os pacientes obesos podem desenvolver macroatelectasias e outras complicações pós-operatórias. Assim, os valores de $\mathrm{PO}_{2}$ e $\mathrm{PCO}_{2}$ estão alterados no primeiro e no terceiro dia pós-operatório, mesmo não tendo diferença significante estatisticamente.

Alguns valores pulmonares são moderadamente reduzidos em pacientes com síndrome de hipoventilação devido primeiramente à uma doença restritiva, ao volume de reserva expiratório marcadamente diminuído, sugestivo de um colapso alveolar e também da derivação, que ocorre durante a expiração e melhoram com a perda de peso ${ }^{13}$.

A dor pós-operatória, bem como o tipo de analgesia e a imobilização prolongada, influenciam para uma possível hipoxemia ${ }^{18}$, sendo que no pós-operatório imediato existe o oferecimento maior de oxigênio ao paciente. Durante a realização deste estudo, foram encontrados no pós-operatório imediato dois aspectos bem marcantes: pacientes em hipóxia acentuada ou com aporte de oxigênio muito superior.

Além da dor e do tipo de analgesia, o tipo de incisão, o tempo cirúrgico, a posição supina, a distensão abdominal favoreceriam à paralisia diafragmática, elevação das cúpulas e ao aparecimento de atelectasias (disfunções diafragmáticas pós-operatórias), que reduziriam em muito o conforto do paciente e a fisioterapia efetiva ${ }^{19}$.

Dentre as complicações respiratórias após as operações do andar superior do abdome estão as microatelectasias ou o colapso alveolar periférico, o tromboembolismo pulmonar, a febre e a tosse produtiva, as alterações quanto ao consumo de oxigênio na indução anestésica, predispondo à infecções pulmonares e à diminuição da capacidade residual funcional, sendo que a otimização do estado respiratório desses pacientes antes da operação, incluindo fisioterapia (mobilização precoce), broncodilatadores, profilaxia antibiótica e a orientação para cessar o tabagismo podem diminuir os riscos pulmonares pós-operatórios ${ }^{4,20,22}$.

Neste estudo, não foram detectadas doenças pulmonares importantes, as espirometrias não detectaram alterações importantes no pré-operatório e a gasometria arterial no préoperatório também não detectou alterações graves, sendo que a alteração na gasometria arterial, somente acontece, comparativamente, no primeiro dia de pós-operatório.

Num período de seis meses, os obesos submetidos ao tratamento cirúrgico, com perda significativa de peso melhoram significativamente o PImáx e o PEmáx em relação ao pré-operatório demonstrando uma melhora no "endurance" muscular respiratório ${ }^{9,23,24}$.

A adoção do incentivador da respiração (Respiron®) no estudo, deveu-se principalmente ao incentivo do exercício respiratório profilático para treinamento e melhora da dispnéia ${ }^{25}$ 
no pré-operatório e como orientação de comparação de melhora após 30 dias de pós-operatório, fazendo-se também esse incentivo até 30 dias. Como descrito anteriormente, com a pontuação adotada não houve melhora significativa nas comparações das médias, salientando somente que o tempo de internação foi de 5,81 dias $( \pm 1,39)$.

No presente estudo $36 \%$ dos pacientes estavam com a cúpula diafragmática direita elevada, provavelmente como conseqüência da esteatose hepática este fato favorece o comprometimento da base pulmonar direita no pós-operatório.

A parede torácica e o diafragma no final da expiração estão alterados, diminuindo a capacidade vital e o volume de reserva expiratória, demonstrando que os outros volumes máximos podem ou não estar alterados, verificados através de espirometria e testes de caminhadas de seis minutos ${ }^{12}$. A idade e a presença ou não do tabagismo nesta população pode influenciar a piora da reserva respiratória ${ }^{26-32}$.

O acompanhamento fisioterápico de orientação préoperatória, o tratamento e a profilaxia fisioterápica respiratória, podem ter figurado como um dos fatores que contribuíram para que os pacientes desse estudo não tivessem complicações respiratórias, sendo que os dados que não obtiveram significância, possivelmente não foram obtidos em virtude do curto período do estudo, ou então por um seguimento menos rigoroso destes pacientes no pós-operatório. Em síntese, se esses mesmos pacientes fossem submetidos a um programa especial de exercícios respiratórios e físicos por um período maior, possivelmente os dados de melhora seriam significativos.

\begin{abstract}
Background: The objective this study was to measure quantitatively those breathing alterations until 30 days postoperative. Method: Breathing evaluations were accomplished before and after bariatric surgery, in morbid obese with IMC greater than $39 \mathrm{~kg} / \mathrm{m}^{2}$, through arterial gasometry, spirometry function, manovacuometry, to motivate breathing and circumferences. They were breathing physiotherapeutic accomplished, orientations and treatment in the postoperative, with comparative data among evaluations in the $1^{\text {st }}, 14^{\text {th }} \cdot e 30^{\text {th }}$ postoperative day. Results: Until the $30^{\text {th }}$ postoperative day, those individuals didn't achieve significant differences in the evaluated parameters, and showed no breathing complications for physiotherapeutic treatment. Conclusion: There were no alterations in the analyzed parameters, nor breathing complications in this study with intervention physiotherapy before and after bariatric surgery. Studies should be accomplished, for a longer postoperative period, including specific exercises to achieve different results.
\end{abstract}

Key words: Physical therapy; Obesity; Bariatric surgery; Spirometry; Respiratory function tests.

\section{REFERÊNCIAS}

1. Bickelmann AG, Burwell CS, Robin ED, Whaley RD. Extreme obesity associated with alveolar hypoventilation-a Pickwickian syndrome. Am J Med. 1956;21(5):811-8.

2. Munsh Y, Sagnard P. Le point de vue de l'anesthésiste dans le traitment chirurgical de l'obésité morbide: traitement chirurgical de l'obésité mordide. Ann Chir. 1997;51(2):183-8.

3. Pereira EDB, Farensin SM, Fernandes ALG. Morbidade respiratória nos pacientes com e sem síndrome obstrutiva submetidos a cirurgia abdominal alta. Rev Assoc Med Bras. 2000;46(1):1522.

4. Ali J, Weisel RD, Layug AB, Kripke BJ, Hechtman HB. Consequences of postoperative alterations in respiratory mechanics. Am J Surg. 1974;128(3):376-82.

5. Charuzi I, Lavie P, Peiser J, Peled R. Bariatric surgery in morbidly obese sleep-apnea patients: short- and long-term follow-up. Am J Clin Nutr. 1992;55(2 Suppl):594S-596S.

6. Hall JC, Tapper J, Tarala R. The cost-efficiency of incentive spirometry after abdominal surgery. Aust.N Z J Surg. 1993;63(5):356-9.

7. Geloneze B, Tambascia MA, Pareja JC, Repetto EM, Magna LA. The insulin tolerance test in morbidly obese patients undergoing bariatric surgery. Obes Res. 2001;9(12):763-9.

8. Rochester DF. Obesity and pulmonary function. In: Alpert MA, Alexander JK, editors. The heart and lung in obesity. New York: Futura Publising; 1998. p.109-31.
9. Weiner P, Waizman J, Weiner M, Rabner M, Magadle R, Zamir D. Influence of excessive weight loss after gastroplasty for morbid obesity on respiratory muscle performance. Thorax. 1998;53(1):39-42.

10. De Lorenzo A, Maiolo C, Mohamed EI, Andreoli A, Petrone-De Luca P, Rossi P. Body composition analysis and changes in airways function in obese adults after hypocaloric diet. Chest. 2001;119(5):1409-15.

11. Ladosky W, Botelho MA, Albuquerque JP Jr. Chest mechanics in morbidly obese non-hypoventilated patients. Respir Med. 2001;95(4):281-6.

12. Ray CS, Sue DY, Bray G, Hansen JE, Wasserman K. Effects of obesity on respiratory function. Am Rev Respir Dis. 1983;128(3):501-6.

13. Sugerman HJ, Fairman RP, Sood RK, Engle K, Wolfe L, Kellum JM. Long-term effects of gastric surgery for treating respiratory insufficiency of obesity. Am J Clin Nutr. 1992;55(2 Suppl):597S$601 \mathrm{~S}$.

14. Jackson CV. Preoperative pulmonary evaluation. Arch Intern Med. 1988;148(10):2120-7.

15. Gudmundsson G, Cerveny M, Shasby DM. Spirometric values in obese individuals. Effects of body position. Am J Respir Crit Care Med. 1997;156(3 Pt 1):998-9.

16. Cullen JH, Formel PF. The respiratory defects in extreme obesity. Am J Med. 1962;32:525-31.

17. Bromberg N, Matuszak JP, Delecluze Ph, Atat I, Prevot JM, Krivosic-Horberor. Fonction pulmonaire aprés cholecystectomie 
par voie laparoscopique chez l'obese. Ann Fr Anesth Reanim. 1991;10(Suppl):R84.

18. Olsén MF, Josefson K, Lönroth H. Chest physiotherapy does not improve the outcome in laparoscopic fundoplication and vertical-banded gastroplasty. Surg Endosc. 1999;13(3):260-3.

19. Simonneau G, Vivien A, Sartene R, Kunstlinger F, Samii K, Noviant Y, Duroux P. Diaphragm dysfunction induced by upper abdominal surgery. Role of postoperative pain. Am Rev Respir Dis. 1983;128(5):899-903.

20. Mason EE, Renquist KE, Jiang D. Perioperative risks and safety of sugery for severe obesity. Am J Clin Nutr. 1992;55(2 Suppl):573S-576S.

21. Goulding ST, Hovell BC. Anaesthetic experience of vertical banded gastroplasty. Br J Anaesth. 1995;75(3):301-6.

22. Kress JP, Pohlman AS, Alverdy J, Hall JB. The impact of morbid obesity on oxigen cost of breathing (V O2RESP) at rest. Am J Respir Crit Care Med. 1999;160(3):883-6.

23. Sahebjami H, Gartside PS. Pulmonary function in obese subjects with a normal FEV1 / FVC ratio. Chest. 1996;110( 6):1425-9.

24. Maniscalco M, Zedda A, Giardiello C, Faraone S, Cerbone MR, Cristiano S, Sofia M. Effect of bariatric surgery on the sixminute walk test in severe uncomplicated obesity. Obes Surg. 2006;16(7):836-41

25. EL-Gamal H, Khayat A, Shikora S, Unterborn JN. Relationship of dyspnea to respiratory drive and pulmonary function tests in obese patients before and after weight loss. Chest. 2005;128(6):3870-4. Comment in: Chest. 2006;130(4):1282-3.

26. Zavorsky GS, Murias JM, Kim do J, Gow J, Sylvestre JL, Christou NV. Waist-to-hip ratio is associated with pulmonary gas exchange in the morbidly obese. Chest. 2007;131(2):362-7.

27. Muzumdar H, Rao M. Pulmonary dysfunction and sleep apnea in morbid obesity. Pediatr Endocrinol Rev. 2006;3(Suppl 4):579-83.
28. Hamoui N, Anthone G, Crookes PF. The value of pulmonary function testing prior to bariatric surgery. Obes Surg. 2006;16(12):1570-3.

29. Arias MA, Alonso-Fernández A, García-Río F. Left ventricular diastolic abnormalities in obese subjects. Chest. 2005;128(6):3870-4. Comment in: Chest. 2006;130(4):12821283.

30. Santana AN, Souza R, Martins AP, Macedo F, Rascovski A, Salge JM. The effect of massive weight loss on pulmonary function of morbid obese patients. Respir Med. 2006;100(6):1100-4. Epub 2005 Oct 21.

31. Hulens M, Vansant G, Claessens AL, Lysens R, Muls E. Predictors of 6-minute walk test results in lean, obese and morbidly obese women. Scand J Med Sci Sports. 2003;13(2):98105.

32. Dávila-Cervantes A, Domínguez-Cherit G, Borunda D, Gamino R, Vargas-Vorackova F, González-Barranco J, Herrera MF. Impact of surgically-induced weight loss on respiratory function: a prospective analysis. Obes Surg. 2004;14(10):1389-92.

Como citar este artigo:

Silva AMO, Boin IFS, Pareja JC, Magna LA. Análise da função respiratória em pacientes obesos submetidos à cirurgia Fobi-Capella. Rev Col Bras Cir. [periódico na Internet] 2007 Set-Out; 34(5). Disponível em URL: www.scielo.br/rcbc

Endereço para correspondência:

Aurea Maria Oliveira da Silva

Rua Dr. Mario de Nucci, 105

Cidade Universitária

13083-290 - Campinas - SP - Brasil

Fone: (55)1932890679/ FAX: (55)1932891860

E-mail: amos@obelix.unicamp.br 